Recepción: 18/02/2019

Aceptación: 22/03/2019

Publicación: 05/05/2019

Artículo de revisión

\title{
El aprendizaje basado en tareas y las competencias comunicativas del inglés en estudiantes universitarios
}

\section{Task-based learning and communicative skills of English in university students}

\section{Aprendizagem baseada em tarefas e habilidades comunicativas de inglês em estudantes universitários}

\author{
Magdalena del Carmen Toala-Alarcón ${ }^{\mathrm{I}}$ \\ mtoala@utm.edu.ec
Nancy Johanna Vivero-Cedeño II nvivero@utm.edu.ec
Miguel Ángel Macías-Loor ${ }^{\text {III }}$ mamacias@utm.edu.ec \\ Oscar Elias Bolívar-Chávez ${ }^{\text {IV }}$ \\ obolivar@utm.edu.ec
}

\section{Correspondencia:mpalma@utm.edu.ec}

I. Magíster en Enseñanza del Idioma Inglés, Licenciada en Ciencias de la Educación Especialidad Inglés, Profesora de Segunda Enseñanza Idiomas y Lingüística Esp. Inglés, Docente del Instituto de Lenguas de la Universidad Técnica de Manabí, Portoviejo, Ecuador.

II. Magíster en Enseñanza del Idioma Inglés, Licenciada en Ciencias de la Educación Especialidad Inglés, Docente del Instituto de Lenguas de la Universidad Técnica de Manabí, Portoviejo, Ecuador.

III. Magíster en Enseñanza del Idioma Inglés, Licenciado en Ciencias de la Educación Especialidad Inglés, Docente del Instituto de Lenguas de la Universidad Técnica de Manabí, Portoviejo, Ecuador.

IV. Ph.D en Educación, Master en Nuevas Tecnologías aplicadas a la Educación, Licenciado en Ciencias de la Educación Especialidad Instrumentistas Pedagogo en Saxofón. CETAC, Docente de la Universidad Técnica de Manabí, Portoviejo, Ecuador. 


\title{
Resumen
}

El presente artículo, se propuso como objetivo caracterizar el aprendizaje basado en tareas y las competencias comunicativas del inglés en estudiantes universitarios. La metodología fue de tipo descriptiva- documental. La población la constituyeron 12 docentes universitarios y el instrumento fue un cuestionario con una escala de 5 alternativas a saber; siempre, casi siempre, algunas veces, casi nunca y nunca. El análisis de la información fue procesado estadísticamente a través del paquete estadístico SPSS versión 19. Entre los resultados se pudo constatar que las competencias socio-lingüísticas, según el 71\% de los docentes investigados casi siempre incluyen para su uso el conocimiento de las reglas socioculturales, de las normas, los estilos y los registros de la lengua que están tratando. Dentro de las conclusiones se pudo determinar que el aprendizaje fundamentado en tareas fortalece las competencias comunicativas del inglés en los estudiantes universitarios.

Palabras clave: Aprendizaje basado en tareas; competencias comunicativas; inglés y estudiantes universitarios.

\begin{abstract}
This article was intended to characterize task-based learning and english communication skills in university students. The methodology was descriptive- documentary. The population was made up of 12 university teachers and the instrument was a questionnaire with a scale of 5 alternatives namely; always, almost always, sometimes, almost never and never. The analysis of the information was statistically processed through the SPSS version 19 statistical package. Among the results it was found that socio-linguistic competencies, according to $71 \%$ of teachers investigated almost always include for use knowledge of sociocultural rules, standards, styles and records of the language that are Trying. Among the findings it was possible that task-based learning strengthens English's communicative skills in university students.
\end{abstract}

Keywords: Task-based learning; communication skills; English and college students 


\section{Resumo}

Este artigo teve como objetivo caracterizar a aprendizagem baseada em tarefas e as competências comunicativas do inglês em estudantes universitários. A metodologia foi descritiva-documental. A população era composta por 12 professores universitários e o instrumento era um questionário com uma escala de 5 alternativas, a saber; sempre, quase sempre, às vezes, quase nunca e nunca. A análise das informações foi processada estatisticamente através do pacote estatístico SPSS versão 19. Entre os resultados, verificou-se que as competências sócio-linguísticas, de acordo com $71 \%$ dos professores investigados, quase sempre incluem pelo uso do conhecimento das regras socioculturais, dos padrões, estilos e registros do idioma com o qual estão lidando. Nas conclusões, determinou-se que a aprendizagem baseada em tarefas reforça as competências comunicativas do inglês em estudantes universitários.

Keywords: Aprendizagem baseada em tarefas; habilidades de comunicação; Estudantes de inglês e universitários.

\section{Introducción}

Una tarea es una actividad que debe permitir la concreción de un objetivo con un resultado específico, donde el énfasis está en el intercambio de significados y no en producir una forma específica del lenguaje. Su propósito fundamental es dar a conocer a partir del lenguaje en un contexto significativo que implique su uso de manera relevante y auténtica. Siendo así, las tareas docentes, vistas como la célula del proceso, son las acciones realizadas por el profesor y los estudiantes, en ciertas circunstancias pedagógicas, con el fin de alcanzar como objetivo elemental: el profesor plantea una situación problemática para que el estudiante resuelva. Atendiendo esta posición, el proceso docente se desarrolla de tarea en tarea lograr el comportamiento esperado por los estudiantes. De modo que todo proceso docente en la educación superior estará dado por una serie sucesiva de situaciones, desde la primera conferencia hasta el trabajo de diploma o de culminación de carrera. Con base a este planteamiento, se propuso como objetivo caracterizar el aprendizaje basado en tareas y las competencias comunicativas del inglés en estudiantes universitarios.

\section{6}

Pol. Con. (Edición núm. 33) Vol. 4, No 5, mayo 2019, pp. 444-451, ISSN: 2550 - 682X 


\section{Desarrollo}

\section{El aprendizaje basado en tareas}

Harmer (2001), indica tres etapas básicas para el desarrollo de las clases basadas en tareas:

- Pre-task o actividad previa a la tarea: donde el profesor explora el tema con los estudiantes e indica las palabras y frases útiles para el desarrollo de la actividad.

- Task cycle o ciclo de tarea o desarrollo: donde los estudiantes completan la tarea en parejas o grupos, mientras el profesor monitorea desde cierta distancia.

- Language focus stage o foco en el lenguaje o interacción: donde el profesor y los estudiantes analizan los aspectos de la lengua que facilitaron o hicieron más compleja la tarea dada por el profesor.

\section{Modelos de aprendizaje por tareas}

Para efectos de este estudio se tomó las propuestas por Estaire (2007), la cual divide en cuatro áreas fundamentales: competencia gramatical, competencia socio-lingüística, competencia discursiva y competencia estratégica.

Competencia gramatical (o lingüística): Incluye conocimiento del léxico, de las reglas de la morfología, la sintaxis, las oraciones gramaticales, la semántica y la fonología y además la habilidad para usar con exactitud la morfología y la sintaxis, la fonología y la semántica de la lengua extranjera. Era este prácticamente el único componente objeto de atención con el enfoque estructural, que antecedió al enfoque comunicativo.

Competencia discursiva: Relacionada con el dominio de cómo combinar las formas gramaticales y el significado de manera tal que se obtenga un texto hablado o escrito, en diferentes tipos de textos. Ella incluye además la habilidad para unir las ideas tanto en el texto oral como en el escrito, lo cual significa el reconocimiento de que la lengua existe por encima del nivel de la oración, o sea, a nivel textual. El tratamiento a esta competencia tiene una importante implicación metodológica: dejar que los estudiantes expresen sus propias ideas y no que digan en otras palabras el contenido que el profesor les dio. 
Competencia socio-lingüística: Incluye el conocimiento de las reglas socioculturales para el uso, el conocimiento de las normas, los estilos y los registros de la lengua que se trate. Se expresa en el uso de la lengua y los elementos paralinguísticos de manera apropiada al contexto en que se desarrolla el acto comunicativo. Significa ofrecer la cantidad necesaria de información, ni muy poca ni mucha; ser relevante de acuerdo con el tópico; y, ser sincero a no ser que sea necesario el uso del sarcasmo. Esta competencia incluye el conocimiento sobre las normas culturales, los estilos y los registros lingüísticos e implica además rebasar la consideración de lo correcto por lo apropiado (González, 2000).

Competencia estratégica: Referida a poseer estrategias para ser utilizadas en caso de insuficiencias en cualquier otra de las áreas de competencia. Consiste en estrategias verbales y no-verbales que pueden entrar en acción cuando existen rupturas en el mensajes o vacíos debido a insuficiencias en la competencia comunicativa en general. Es la habilidad para comenzar, continuar, enfatizar, concluir, etc. un acto comunicativo.

La fluidez y la exactitud, son por lo general lo más considerado en el marco de un enfoque comunicativo, por un lado, para facilitar y dar rapidez a la elaboración de los textos y por el otro permitir mayor perfección de los elementos fónicos, léxico-semánticos y morfosintácticos de la lengua. (Acosta, 2002).

\section{Competencias comunicativas del Idioma del Ingles}

Las competencias consideradas, fueron las presentadas por Rivera (2001), las cuales establecen tres competencias a saber:

\section{Competencia lingüística:}

Se refiere al conocimiento de los recursos formales de la lengua como sistema y a la capacidad para utilizarlos en la formulación de mensajes bien formados y significativos. Incluye los conocimientos y las destrezas léxicas, fonológicas, sintácticas y ortográficas, entre otras. Esta competencia implica, no sólo el manejo teórico de conceptos gramaticales, ortográficos o semánticos, sino su aplicación en diversas situaciones.

\section{8}

Pol. Con. (Edición núm. 33) Vol. 4, No 5, mayo 2019, pp. 444-451, ISSN: 2550 - 682X 


\section{Competencia pragmática:}

Se relaciona con el uso funcional de los recursos lingüísticos y comprende, en primer lugar, una competencia discursiva que se refiere a la capacidad de organizar las oraciones en secuencias para producir fragmentos textuales. En segundo lugar, implica una competencia funcional para conocer, tanto las formas lingüísticas y sus funciones, como el modo en que se encadenan unas con otras en situaciones comunicativas reales.

\section{Competencia sociolingüística:}

Se refiere al conocimiento de las condiciones sociales y culturales que están implícitas en el uso de la lengua. Por ejemplo, se emplea para manejar normas de cortesía y otras reglas que ordenan las relaciones entre generaciones, géneros, clases y grupos sociales. También se maneja al entrar en contacto con expresiones de la sabiduría popular o con las diferencias de registro, de dialecto y de acento.

\section{Metodología}

La metodología fue de tipo descriptiva- documental, que según Hurtado (2015), se refiere a la investigación que tiene como objetivo analizar un evento y comprenderlo en términos de sus aspectos menos evidentes. El cuanto al enfoque documental refiere a la revisión teórica del aprendizaje basado en tareas y las competencias comunicativas del inglés en estudiantes universitarios. La población la constituyeron 12 docentes universitarios y el instrumento fue un cuestionario con una escala de 5 alternativas a saber; siempre, casi siempre, algunas veces, casi nunca y nunca. El análisis de la información fue procesado estadísticamente a través del paquete estadístico SPSS versión 19. 


\section{Resultados}

Dentro de los resultados con mayor relevancia para la investigación, se pueden destacar:

Se constató que el 55\% de los docentes investigados, coincidieron en opinar que siempre en sus tareas incluyen el conocimiento del léxico y las oraciones gramaticales y prestan muy poca atención a los enfoques de tipo estructural.

Para el $66 \%$ de los investigados, casi siempre logran en sus tareas relacionar las formas gramaticales y su significado, para obtener un texto hablado o escrito, obteniendo unir las ideas tanto en el texto oral como en el escrito, para corroborar que la lengua priva el nivel de la oración.

Las competencias socio-lingüísticas, según el $71 \%$ de los docentes investigados algunas veces, incluyen para su uso el conocimiento de las reglas socioculturales, de las normas, los estilos y los registros de la lengua que están tratando.

Solo el $45 \%$ de los investigados, consideraron que algunas veces utilizan las competencias estratégicas verbales y no-verbales que pueden entrar en acción cuando existen rupturas en el mensaje durante la competencia comunicativa.

Se evidencio en el 53\% de los docentes investigados, que casi siempre utilizan la competencia lingüística para conseguir mensajes bien formados y significativos, que se ajusten a los preceptos teóricos que lo sustentan.

En el $63 \%$ de los investigados, se pudo constatar que algunas veces hacen uso de la competencia pragmática para lograr el uso funcional de los recursos lingüísticos para lograr organizar las oraciones para producir fragmentos textuales y las competencias sociolingüísticas, casi siempre según el 69\% de los investigados las han utilizados para considerarlas como tareas durante el aprendizaje del idioma Ingles. 


\section{Conclusiones}

- El estudio de relación entre las dos variables, permitió determinar que el aprendizaje fundamentado en tareas fortalece las competencias comunicativas del inglés en los estudiantes universitarios.

- La variable aprendizaje basado en las tareas, casi siempre es utilizada como actividad previa a la tarea, para completar tareas en grupos ni en los procesos de interacción docente -alumno.

- Se constató que, dentro de las competencias comunicativas del inglés en estudiantes universitarios, los docentes casi siempre utilizan las competencias lingüísticas, pragmáticas y sociolingüistas, presentando menor uso las de tipo lingüística.

\section{Referencias Bibliográficas}

González Rey, F. (2000). La Comunicación Educativa: Su manejo en la institución. Soporte magnético. La Habana. ISP "EJ Varona”.

Acosta, R (2002). Communicative Language Teaching. (CD Carr era de Lengua Inglesa. MINED. Primera Versión).

Rivera, J. (2001). Modelo Teórico de la enseñanza sistémico-comunicativa para el desarrollo de la habilidad de comprensión de lectura en inglés en el nivel medio (superior). Tesis Doctoral. ICCP. La Habana. Cuba.

Hurtado, J. (2015). El Proyecto de Investigación. Octava Edición. Caracas. Venezuela.

Estaire, S. (2007). La enseñanza de lenguas mediante tareas: principios y planificación de unidades didácticas. 